\title{
Impact of Histological and Molecular Parameters on Prognosis of Oral Squamous Cell Carcinoma: Analysis of 290 Cases
}

\author{
B. S. M. S. Siriwardena $\mathbb{D}^{1,2}$ H. D. N. U. Karunathilaka $\mathbb{D}^{2}{ }^{2}$ P. V. R. Kumarasiri $\mathbb{D},^{3}$ \\ and W. M. Tilakaratne $\mathbb{D}^{1,4}$ \\ ${ }^{1}$ Department of Oral Pathology, Faculty of Dental Sciences, University of Peradeniya, Sri Lanka \\ ${ }^{2}$ Centre for Research in Oral Cancer, University of Peradeniya, Sri Lanka \\ ${ }^{3}$ Department of Community Medicine, Faculty of Medicine, University of Peradeniya, Sri Lanka \\ ${ }^{4}$ Department of Oral and Maxillofacial Clinical Sciences, Faculty of Dentistry, University of Malaya, Malaysia
}

Correspondence should be addressed to B. S. M. S. Siriwardena; samadarani@yahoo.com

Received 12 July 2020; Accepted 25 September 2020; Published 15 October 2020

Academic Editor: Taiyoun Rhim

Copyright ( 2020 B. S. M. S. Siriwardena et al. This is an open access article distributed under the Creative Commons Attribution License, which permits unrestricted use, distribution, and reproduction in any medium, provided the original work is properly cited.

\begin{abstract}
Background. Nodal metastasis is a critical factor in predicting the prognosis of oral squamous cell carcinoma (OSCC). When patients present with a clinically positive neck, the treatment of choice is radical neck dissection. However, management of a clinically negative neck is still a subject of significant controversy. Aim. This study was carried out in order to propose a model to predict regional lymph node metastasis of OSCC using histological parameters such as tumour stage, tumour size, pattern of invasion (POI), differentiation of tumour, and host immune response, together with the expression levels of six biomarkers (periostin, HIF-1 $\alpha$, MMP-9, $\beta$-catenin, VEGF-C, and EGFR), and, furthermore, to compare the impact of all these parameters on recurrence and $3 \mathrm{yr}$ and $5 \mathrm{yr}$ survival rates. Materials and Method. Histological materials collected from the archives were used to evaluate histological parameters and immunohistochemical profiles. Standard methods were used for immunohistochemistry and for evaluation of results. Data related to recurrence and survival (3 and 5 years) was also recorded. Clinical data was collected from patients' records. Results. Male to female ratio was $3: 1$. The commonest site of OSCC was the buccal mucosa, and majority of them were T3 or T4 tumours presented at stage $4.62 .5 \%$ of the tumours were well differentiated. Three-year and 5-year survival rates were significantly associated with lymph node metastasis and recurrence. POI was significantly correlated with tumour size, stage, 3-year survival, EGFR, HIF-1 $\alpha$, periostin, and MMP-9 $(p<0.05)$. Expression of EGFR showed a direct association with metastasis $(p<0.05)$. Conclusion. POI, level of differentiation, and expression of EGFR are independent prognostic markers for lymph node metastasis. Therefore, these parameters may help in treatment planning of a clinically negative neck.
\end{abstract}

\section{Introduction}

Oral cancer is the commonest cancer in men in Sri Lanka, and 3-4 people die every day due to the disease [1]. Nodal metastasis is a significant prognostic indicator. When patients present with a clinically positive neck, the treatment of choice is excision of the tumour with radical neck dissection (RND), whilst the management of a clinically negative neck is still controversial. Usually, for the latter, either elective neck dissection (END) or "watchful-waiting" policy is practised [2]. Although END could help in eliminating undetectable metastasis, majority of the patients may not need such radical treatment, as it is associated with significant morbidity. In addition, it removes the natural barrier, the lymphatic system $[3,4]$.

Size and primary site of the tumour, TNM stage, and some histopathological parameters have a significant impact on prognosis. Tumour differentiation, pattern of invasion, tumour thickness, host immune response, and vascular and perineural invasion are some of the proven parameters. We 
and others have demonstrated with previous studies that the pattern of invasion is a very reliable factor in predicting metastasis [5-7].

Invasive front or the tumour-host interface of the cancer is known to be important in predicting metastasis and prognosis [8]. Many studies have proven that the location of molecular markers at the invasive front is more important than assessment of their expression in the whole tumour [9]. The number of studies assessing the invasive front using molecular markers appeared to be inadequate to draw scientifically valid conclusions to manage patients [3]. Therefore, analysis of the expression levels of various molecules at the invasive front in a larger sample of patients is a necessity to identify the markers that predict metastasis and prognosis.

In our previous studies, we have shown that there is a significant expression of molecular markers such as hypoxiainducible factor- $1 \alpha$ (HIF- $1 \alpha)[10,11]$, periostin [12], matrix metalloproteinase-9 (MMP-9) [13], vascular endothelial growth factor-C (VEGF-C) $[14,15]$, epidermal growth factor receptor (EGFR) [16], and $\beta$-catenin [16] in OSCC and their ability to serve as potential molecules for predicting metastasis. Further, it has been stated that the use of molecular markers, together with traditional histological parameters, improves comprehensive management of patients with OSCC [17]. This may provide a better prediction of prognosis and proper guidance for management of neck nodes. This will invariably reduce the morbidity and cost of advanced treatment procedures.

Although the significance of many of the biomarkers in OSCC has been reported, large prospective studies integrating the most reliable predictive biomarkers are crucial to control the variability and for definite clarification of the prognostic significance of some of these molecular markers [17]. Therefore, the present study is aimed at investigating the expression of the abovementioned molecular markers at the invasive front and at comparing their relationship with histological parameters, metastasis, and survival. This has not been widely studied using a large number of patients. We hypothesize that the above markers in different combinations and selected histological parameters may have a role in predicting metastasis, hence the survival.

Therefore, our aim was to establish a prediction model combining histopathological parameters with a few selected molecular markers.

\section{Materials and Methods}

2.1. Case Selection. Histologically confirmed OSCCs treated with excision of the lesion and neck dissection at different oral and maxillofacial units of Sri Lanka from January 1999 to December 2013 were selected for the study. Demographic and clinical data were gathered from clinical records of patients, and histological information such as tumour differentiation and nodal status were obtained from the oral pathology database. Survival and recurrence data were also collected.

2.2. Histopathological Assessment. Paraffin-embedded tissue blocks and slides of the primary tumours and the metastatic nodes were retrieved. The best representative section from the main tumour was selected to assess the pattern of invasion, and the same paraffin block from all cases was used to take sections for immunohistochemical staining. First and last slides were stained with haematoxylin and eosin to confirm the presence of tumour. Data pertaining to nodal status was recorded for each level. The cases with inadequate data were excluded from the study.

2.3. Immunohistochemistry (IHC). The sections were placed on APS-coated slides. The tissue sections were deparaffinized and rehydrated in a graded series of alcohols. Endogenous peroxidase activity was blocked with $0.3 \% \mathrm{H}_{2} \mathrm{O}_{2}$ in methanol for 30 minutes. All selected cases were stained by the EnVision+ system using primary antibodies for EGFR [18], $\beta$ catenin [19], VEGF-C [14], MMP-9 [20], periostin [12], and HIF- $1 \alpha[11]$. Incubation time for each antibody is stated in Table 1.

The immunohistochemical staining was evaluated with a grading system according to the intensity of staining. Staining was recorded for each antibody on each case independently, especially at the advancing front (Table 2).

2.4. Statistical Analysis. Statistical analysis was performed using SPSS for Windows version 16. Data was analysed using descriptive statistics. Pearson's chi-square test was used to compare proportions. The statistical significance was accepted at $p<0.05$. Logistic regression was used to determine a model for metastasis of OSCC with the histological parameters and molecular biomarkers.

\section{Results}

\subsection{Demographic Data}

3.1.1. Sex. Total of 290 cases were selected for the study. Of them, 218 were males and 72 were females (male:female, $3: 1$ ) and age ranges from 31 to 85 years. Majority of the patients were in the 51-60-year age group (34.8\%). The commonest site was the buccal mucosa for both males and females ( $45 \%$ and $44.4 \%$, respectively). However, majority of tongue cancers were in the 31-40-year age group (54\%). Most tumours were at T3 (48.6\%) and stage $4(57.1 \%)$ irrespective of the gender.

Both males (49.5\%) and females (52.8\%) had mainly well-differentiated SCC. Moderate host immune response was commonly seen in both genders $(47.7 \%$ in males and $48.6 \%$ in females). However, most OSCCs with dense host immune response of $29.2 \%$ were recorded among females. Pattern of invasion type IV was found to be the commonest among males with $40.8 \%$, and it was $37.5 \%$ in females.

The overall 3-year and 5-year survival rates were 55.1\% and $37.3 \%$, respectively. Three-year survival rate for males was $52.8 \%$ whilst it was $62.8 \%$ for females. Five-year survival was $38.6 \%$ and $31.8 \%$ in males and females, respectively. $59 \%$ of the males and $60.9 \%$ of the females had recurrences. Out of the total sample, $36.5 \%$ had nodal metastasis although there was a slight preponderance towards females (38.9\%). 
TABLE 1: List of antibodies used.

\begin{tabular}{|c|c|c|c|c|}
\hline Antibody & Species/clonality & Dilution & Incubation time & Source \\
\hline Anti-EGFR antibody clone DAK-H1-WT code M7298 & $\begin{array}{c}\text { Monoclonal mouse antihuman wild-type } \\
\text { EGFR }\end{array}$ & $1: 200$ & $1 \mathrm{hr}$ at R.T & Dako \\
\hline $\begin{array}{l}\text { Anti- } \beta \text {-catenin antibody clone } \beta \text {-catenin- } 1 \text { code } \\
\text { M3539 }\end{array}$ & Monoclonal mouse antihuman $\beta$-catenin & $1: 50$ & $1 \mathrm{hr}$ at R.T & Dako \\
\hline Antiperiostin ab14041 & Rabbit polyclonal to periostin & $1: 450$ & $\begin{array}{l}\text { Overnight at } \\
\qquad 4^{\circ} \mathrm{C}\end{array}$ & Abcam \\
\hline Anti-HIF-1 $\alpha$ [1A3] ab113642 & Mouse monoclonal [1A3] to HIF-1 $\alpha$ & $1: 400$ & $1 \mathrm{hr}$ at R.T & Abcam \\
\hline Anti-VEGFC ab135506 & Rabbit polyclonal to VEGFC & $1: 100$ & $1 \mathrm{hr}$ at R.T & Abcam \\
\hline Anti-MMP-9 [EP1255Y] & Rabbit monoclonal [EP1255Y] to MMP-9 & $1: 300$ & $2 \mathrm{hrs}$ at R.T & Abcam \\
\hline
\end{tabular}

3.1.2. Age. There was no significant gender difference or primary site of occurrence for different age groups, except that SCC in the tongue was common in younger age groups (31-40 years) accounting for $54 \%$ of the total sample. T1 and T2 tumours were mainly seen in the younger group of patients. Among all age groups, majority of the tumours were T3 and stage 4 except in the 31-40-year age group where stage $3(74.5 \%)$ was the commonest. Predominant POI was type IV and the 31-40-year category had the highest percentage of POI type IV (45.5\%). Highest 3-year survival rate of $56.8 \%$ was found in the 31-40-year age group indicating younger patients had a better 3-year survival. In contrast, 5year survival was better in the 51-60-year group (51.4\%), and all other groups were below 50\%. More recurrences were recorded in the 31-40-year group (75.0\%), and the same group had higher metastasis (45.5\%). Therefore, it was obvious that lymph node metastasis was higher in the younger ( $<40$ years) age group.

3.1.3. Primary Site of OSCC. Buccal mucosa (BM) was the most common primary site (44.8\%); hence, most T4 tumours were from the BM. However, majority of the stage 4 tumours were in the lower alveolar ridge (72.2\%). Most welldifferentiated tumours and tumours with dense immune response were from buccal mucosa while the highest percentage of poorly differentiated tumours $(10.9 \%)$ was recorded from the tongue.

Majority of lower alveolar ridge SCC had the highest percentage of lymph node metastasis $(46.2 \%)$ followed by the tongue $(41.1 \%)$ while buccal mucosa had the highest number $(90,69.2 \%)$ of metastasis-negative cases. POI type IV was mainly identified in tongue cancers $(65.8 \%)$ and least in $\mathrm{BM}$ cancers with low metastatic potential. A statistically significant association was identified between the primary site and POI (chi-square value $36.9, p<0.05$ ). $58.8 \%$ of the tongue cancer patients survived less than 3 years. The highest percentage of patients who survived $>3$ years was when the tumour was present on the buccal mucosa and lower alveolar ridge $(61.5 \%)$. However, the results were not statistically significant. Similarly, the poorest 5 -year survival rate was observed from tongue SCC (21.4\%). Tumours in the buccal mucosa recorded the highest 5-year survival out of all primary sites $(42.9 \%)$.
3.1.4. Size and Stage of Tumour. Out of the total sample, T3 tumours were $43.8 \%$ and T1 tumours were only $2.3 \%$. Size of the tumour was not available in 160 cases. Although T1 and T2 tumours were mainly seen in the younger group, the results were statistically not significant. Majority of stage 4 tumours $(61.1 \%)$ were T4. A statistically significant association was observed between these 2 variables $(p<0.05)$. The relationship between tumour size with POI $(p<0.05)$ and nodal metastasis $(p<0.05)$ was also found to be significant statistically.

$57.7 \%$ of the cases were in stage 4 and $5.2 \%$ in stage 1 (data was available in 194 cases). Combination of stages 3 and 4 was almost $80 \%$ as most patients seek their treatment at the late stage leading to difficult management and poor prognosis. A statistically significant association was revealed between tumour stage and host immune response (chisquare value $13, p<0.05)$. Stage of the tumour and POI also showed a statistically significant association (chi-square value $18.9, p<0.05)$. When metastasis was compared with tumour stage, most of the nodal-positive cases $(72.7 \%)$ were from stage 4 (chi-square value 10.0, $p<0.05$ ).

\subsection{Histopathological Parameters}

3.2.1. Host Immune Response. Host response [5, 8] was moderate in half of the cases (47.9\%), and only $7.9 \%$ of the cases had no host response. Significant number of lower alveolar ridge tumours (19.2\%) showed no host response at all. Least number of lymph node metastasis was identified in the dense response group (chi-square value 8.8, $p<0.05$ ). Patients' with dense host response significantly survived for 3 years (72.7\%) (chi-square value is 9.7, $p<0.05$ ). Survival data was available in 15 cases in the minimal host response category, and survival rate was $26.7 \%$. No significant relationship was observed between 5 -year survival rate and host response. Host immune response is an important variable in prognosis of OSCC and included in the logistic regression model to identify which variable affects metastasis of OSCC.

3.2.2. Differentiation of the Tumour. Well and moderately differentiated tumours were the majority (91\%) whilst poorly differentiated tumours were the least (5.5\%). Out of the total sample, $3.4 \%$ of the cases were early invasive squamous cell carcinomas. $62.5 \%$ of the nodal-negative cases were well- 
TABLE 2: Evaluation of immunohistochemistry.

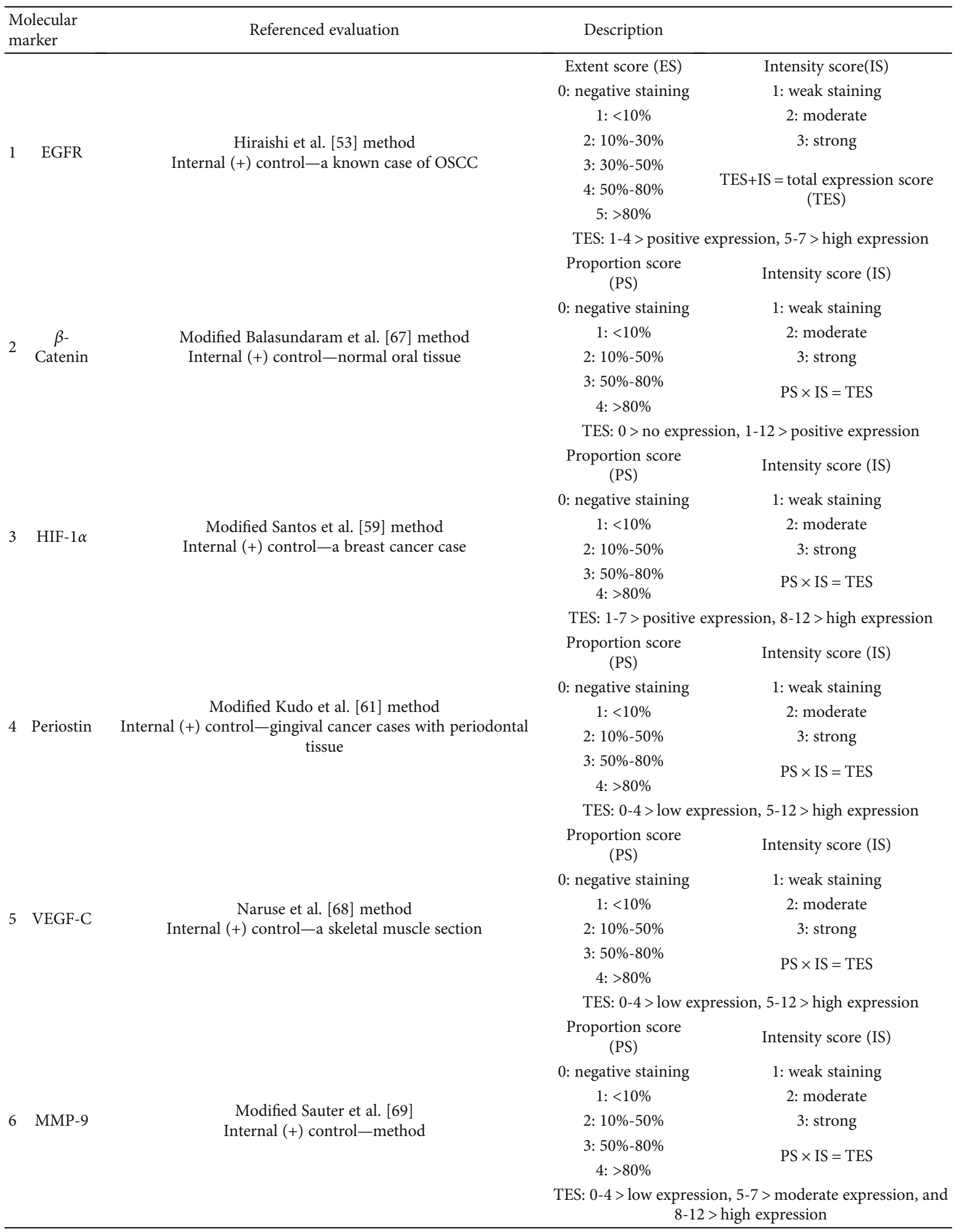


differentiated tumours whilst $68.8 \%$ of the poorly differentiated OSCCs revealed nodal metastasis (chi-square value $32.4, p<0.05$ ). Differentiation of the tumour is an important variable in predicting prognosis of OSCC, and it was included in the logistic regression model.

3.2.3. Nodal Involvement. Lymph node metastasis is one of the major prognostic indicators in OSCC. In the present sample, majority of the cases presented without metastasis (63.4\%). Out of the nodal-positive cases, $52.8 \%$ showed extracapsular invasion.

Nodal involvement was categorized as negative, positive without extra capsular invasion, and positive with extracapsular invasion. Most cases with extracapsular invasion showed POI type IV (58.0\%). Similarly, $50 \%$ of capsular invasion cases also had POI type IV. OSCCs that showed POI type II had very low metastatic rate $(15.4 \%)$ and were significant (chi-square value 24.3, $p<0.05$ ). Nodal metastasis and POI also showed a significant relationship (chi-square value $23.2, p<0.05)$. Further, a statistically significant association was observed between tumour size, stage, host response, tumour differentiation, and EGFR expression with lymph node metastasis $(p<0.05)$ (Table 3 ).

A statistically significant relationship was found between metastasis and 3-year survival. $66.7 \%$ of nodal-negative cases survived for 3 years whilst $33.3 \%$ survived for 3 years when lymph nodes were positive $(p<0.001)$. Similarly, of the patients with positive nodes, $15.4 \%$ survived for 5 years, and in the negative node group, $50 \%$ survived for 5 years $(p<0.001)$. Patients who did not have metastasis had higher survival, and it appears that survival depends on regional lymph node metastasis.

3.2.4. Pattern of Invasion. Pattern of invasion (POI) is a significant predictor of lymph node metastasis. There are 4 grades depending on arrangement of tumour cells at the advancing front ranging from I to IV [5]. Patterns IV (40\%) and III $(33.1 \%)$ were more common than pattern II. There were no cases of pattern I in the sample.

A statistically significant relationship between 3 -year survival and POI was observed (chi-square value 17.7, $p<0.05$ ). $66.1 \%$ of the sample without 3 -year survival had POI type IV. Similarly, $68.1 \%$ of the patients with POI type IV have not survived for 5 years, and the results were significant statistically (chi-square value 25.3, $p<0.05$ ). Majority of the recurred tumours had POI type IV (54.8\%) (chi-square value is $7.7, p<0.05)$. Furthermore, EGFR, MMP-9, HIF- $1 \alpha$, and periostin showed a positive correlation with POI. It is evident that POI is extremely important in prognosis of OSCC; hence, it was included in the logistic regression model as a variable affecting metastasis.

3.3. Tumour Recurrence. Data related to recurrence were available in 123 cases, and 59.3\% had local recurrences. Between 5-year survival and recurrence, a statistically significant association was revealed. $85.4 \%$ of the cases with 5 -year survival had no recurrence, and $77.9 \%$ of the patients without 5 -year survival were presented with recurrence of tumour $(p<0.05)$.

\subsection{Immunohistochemical Markers}

3.4.1. EGFR. EGFR expression can be detected in the cell membrane and cytoplasm of tumour cells (Figure 1). Immunohistochemically, intense expression of EGFR was observed in $78.3 \%$ of the cases, and majority of them had POI type IV while majority of weak expression cases showed POI type II (chi-square value 36.8, $p<0.05$ ). Furthermore, a significant relationship with metastasis was also observed $(p<0.003)$.

3.4.2. $\beta$-Catenin. $\beta$-Catenin expression was detected in the cytoplasm of normal cells and not in cancer cells and was not expressed in majority of the cases (206 out of 290). Only $29.0 \%$ of the cases had a positive expression. Majority of POI type IV showed negative $\beta$-catenin expression. Although the results are not significant, more metastasis was seen in the negative $\beta$-catenin expression group.

3.4.3. HIF-1 $\alpha$. Nuclear and cytoplasmic expression of HIF-1 $\alpha$ was detected in tumour cells. $75.2 \%$ of cases showed strong expression. A significant correlation was found between HIF- $1 \alpha$ and POI type IV suggesting a relationship with poor prognosis (chi-square value $7.8, p<0.05$ ).

3.4.4. Periostin. Periostin expression was detected in the cytoplasm of cancer cells in all cases. Intense staining was observed in $64.5 \%$. Out of highly expressed tumours, $43.9 \%$ had POI type IV and was statistically significant (chi-square value $7.0, p<0.05)$.

3.4.5. VEGF-C. $36.9 \%$ of cases showed intense membranous and cytoplasmic positivity for VEGF-C. Majority (45.8\%) of the intense VEGF-C expression cases had POI type IV. Most of the metastasis-negative cases (61.9\%) had a low expression of VEGF-C.

3.4.6. MMP-9. Strong expression of MMP-9 was detected in $40.2 \%$, and the rest had low expression. A statistically significant association was found between MMP-9 and POI (chisquare value $12.8, p<0.05)$.

3.5. Logistic Regression. A logistic regression statistical model was used to identify the risk factors which are independently responsible for the occurrence of metastasis among the patients. Based on the results of the univariate analysis tumour stage, tumour size, host response, differentiation, POI, and EGFR expression were fitted to the statistical model.

The final regression model identified host immune response, tumour differentiation, and POI as the risk variables which are independently responsible for the occurrence of metastasis. Within the POI, pattern with individual tumour cells (IV) is most significantly associated with the occurrence of metastasis compared to the POI type II (Table 4).

\section{Discussion}

According to the $\mathrm{WHO}$, there is an estimated 657,000 new cases of cancers of the oral cavity and pharynx each year, and more than 330,000 deaths are reported worldwide. The 
TABLE 3: Summary data of the univariate analysis with regard to metastasis.

\begin{tabular}{|c|c|c|c|c|c|}
\hline \multirow{2}{*}{ Variable } & & \multicolumn{2}{|c|}{ Metastasis } & \multirow{2}{*}{ Total (\%) } & \multirow{2}{*}{$p^{*}$ value } \\
\hline & & Positive (\%) & Negative (\%) & & \\
\hline \multirow{6}{*}{ Age } & $31-39$ yrs & $5(4.7)$ & $6(3.3)$ & $11(3.8)$ & \multirow{5}{*}{0.73} \\
\hline & $40-49$ yrs & $17(16.0)$ & $30(16.3)$ & $47(16.2)$ & \\
\hline & $50-59$ yrs & $40(37.7)$ & $61(33.2)$ & $101(34.8)$ & \\
\hline & $60-69$ yrs & $34(32.1)$ & $58(31.5)$ & $92(31.7)$ & \\
\hline & $\geq 70$ yrs & $10(9.4)$ & $29(15.7)$ & $39(13.4)$ & \\
\hline & Total & $106(100.0)$ & $184(100.0)$ & $290(100.0)$ & \multirow{4}{*}{0.635} \\
\hline \multirow{3}{*}{ Gender } & Female & $28(26.4)$ & $44(23.9)$ & $72(24.8)$ & \\
\hline & Male & $78(73.6)$ & $140(76.1)$ & $218(75.2)$ & \\
\hline & Total & $106(100.0)$ & $184(100.0)$ & $290(100.0)$ & \\
\hline \multirow{6}{*}{ Primary site } & Buccal mucosa & $40(37.7)$ & $90(48.9)$ & $130(44.8)$ & \multirow{6}{*}{0.285} \\
\hline & Tongue & $30(28.3)$ & $43(23.4)$ & $73(25.2)$ & \\
\hline & lower alveolar ridge & $12(11.3)$ & $14(7.6)$ & $26(9.0)$ & \\
\hline & Floor of mouth & $7(6.6)$ & $16(8.7)$ & $23(7.9)$ & \\
\hline & Other & $17(16.0)$ & $21(11.4)$ & $38(13.1)$ & \\
\hline & Total & $106(100.0)$ & $184(100.0)$ & $290(100.0)$ & \\
\hline \multirow{3}{*}{ Tumour stage } & 1 and 2 & $18(27.3)$ & $64(50.0)$ & $82(42.3)$ & \multirow{3}{*}{$0.002^{*}$} \\
\hline & 3 and 4 & $48(72.7)$ & $64(50.0)$ & $112(57.7)$ & \\
\hline & Total & $66(100.0)$ & $128(100.0)$ & $194(100.0)$ & \\
\hline \multirow{3}{*}{ Tumour size } & $\mathrm{T} 1, \mathrm{~T} 2$ & $27(48.2)$ & $56(75.7)$ & $83(63.8)$ & \multirow{2}{*}{$0.001^{*}$} \\
\hline & T3, T4 & $29(51.8)$ & $18(24.3)$ & $47(36.2)$ & \\
\hline & Total & $56(100.0)$ & $74(100.0)$ & $130(100.0)$ & \multirow{4}{*}{$0.01^{*}$} \\
\hline \multirow{3}{*}{ Host response } & Dense & $15(14.2)$ & $50(27.2)$ & $65(22.4)$ & \\
\hline & Light & $91(85.8)$ & $134(72.8)$ & $225(77.6)$ & \\
\hline & Total & $106(100.0)$ & $184(100.0)$ & $290(100.0)$ & \\
\hline \multirow{3}{*}{ Differentiation } & Well & $31(29.2)$ & $115(62.5)$ & $146(50.3)$ & \multirow{2}{*}{$<0.001^{*}$} \\
\hline & Poor & $75(70.8)$ & $69(37.5)$ & $144(49.7)$ & \\
\hline & Total & $106(100.0)$ & $184(100.0)$ & $290(100.0)$ & \multirow{5}{*}{$<0.001^{*}$} \\
\hline \multirow{4}{*}{ Pattern of invasion } & POI II & $12(11.3)$ & $66(35.9)$ & $78(26.9)$ & \\
\hline & POI III & $37(34.9)$ & $59(32.1)$ & $96(33.1)$ & \\
\hline & POI IV & $57(53.8)$ & $59(32.1)$ & $116(40.0)$ & \\
\hline & Total & $106(100.0)$ & $184(100.0)$ & $290(100.0)$ & \\
\hline \multirow{3}{*}{ EGFR expression } & Strongly positive & $93(87.7)$ & $134(72.8)$ & $227(78.3)$ & \multirow{3}{*}{$0.003^{*}$} \\
\hline & Weakly positive & 13(12.3) & $50(27.2)$ & $63(21.7)$ & \\
\hline & Total & $106(100.0)$ & $184(100.0)$ & $290(100.0)$ & \\
\hline & No & $77(72.6)$ & $129(70.1)$ & $206(71.0)$ & 0647 \\
\hline$\beta$-Catenin & Yes & $29(27.4)$ & 55 (29.9) & $84(29.0)$ & 0.047 \\
\hline & Total & $106(100.0)$ & $184(100.0)$ & $290(100.0)$ & \\
\hline & Positive & $24(22.6)$ & $48(26.1)$ & $72(24.8)$ & 0513 \\
\hline HIF- $1 \alpha$ & High & $82(77.4)$ & $136(73.9)$ & $218(75.2)$ & 0.515 \\
\hline & Total & $106(100.0)$ & $184(100.0)$ & $290(100.0)$ & \\
\hline & Low & $38(35.8)$ & $65(35.3)$ & $103(35.5)$ & 0920 \\
\hline Periostin & High & $68(64.2)$ & $119(64.7)$ & $187(64.5)$ & 0.929 \\
\hline & Total & $106(100.0)$ & $184(100.0)$ & $290(100.0)$ & \\
\hline & Low & $69(65.1)$ & $114(62.0)$ & $183(63.1)$ & 0.594 \\
\hline VEGF-C & High & $37(34.9)$ & $70(38.0)$ & $107(36.9)$ & 0.394 \\
\hline & Total & $106(100.0)$ & $184(100.0)$ & $290(100.0)$ & \\
\hline MMP-9 & Low & $61(57.5)$ & $112(60.9)$ & $173(59.7)$ & 0.579 \\
\hline
\end{tabular}


TABLE 3: Continued.

\begin{tabular}{lcccc}
\hline \multirow{2}{*}{ Variable } & & \multicolumn{2}{c}{ Metastasis } & Total (\%) \\
\hline & Positive (\%) & Negative (\%) & palue \\
& High & $45(42.5)$ & $72(39.1)$ & $117(40.3)$ \\
& Total & $106(100.0)$ & $184(100.0)$ & $290(100.0)$ \\
\hline
\end{tabular}

* Statistically significant.

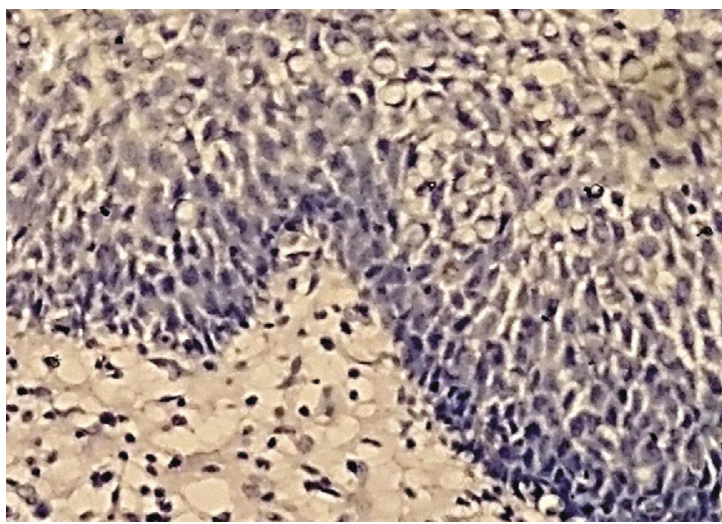

(a)

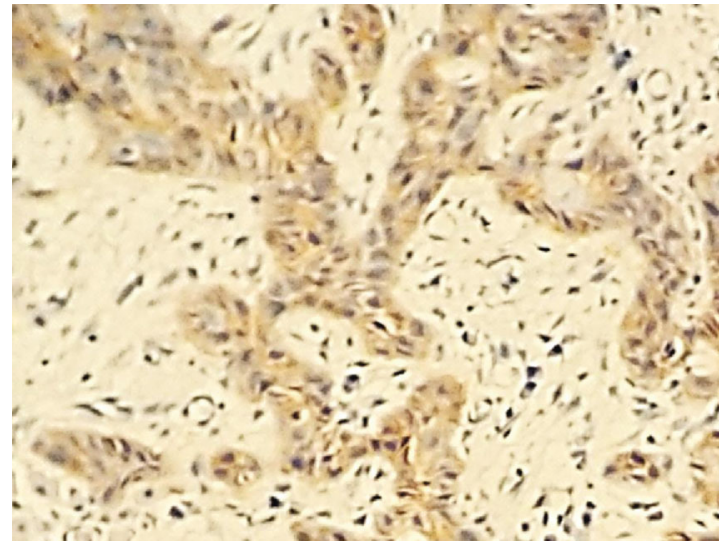

(c)

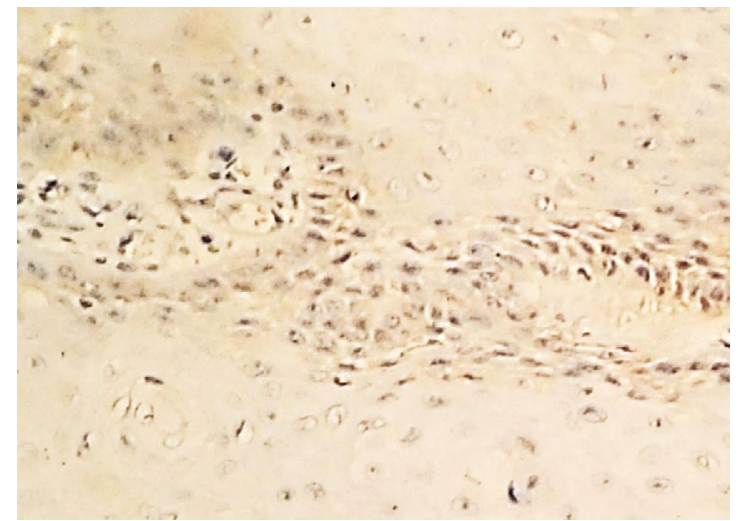

(b)

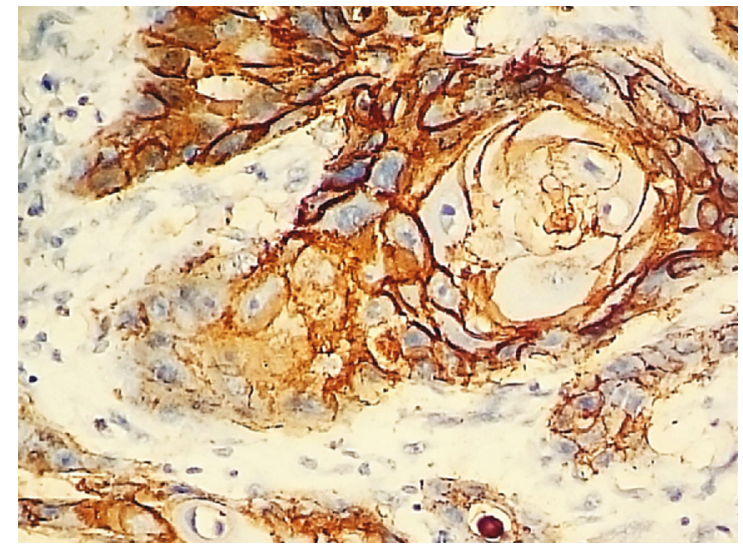

(d)

FIGURE 1: Expression of EGFR in the present study sample. (a) Negative staining of EGFR in normal epithelium. (b) Weak staining of EGFR in OSCC. (c) Moderate staining of EGFR in OSCC. (d) Strong staining of EGFR in OSCC.

TABLE 4: Logistic regression model.

\begin{tabular}{lcccc}
\hline Variables & $B$ & SE & $p$ & Odds ratio (CI) \\
\hline $\begin{array}{l}\text { Tumour differentiation } \\
\text { Inversion pattern }\end{array}$ & 1.304 & 0.51 & 0.10 & $3.68(1.36-9.93)$ \\
$\quad$ & & & & \\
$\quad$ POI II (ref) & & & & 1 \\
$\quad$ POI III & 1.086 & 0.59 & 0.06 & $2.96(0.93-9.41)$ \\
$\quad$ POI IV & 1.760 & 0.70 & 0.12 & $5.81(1.46-23.09)$ \\
Constant & -3.158 & 0.88 & $<0.00$ & \\
\hline
\end{tabular}

burden is particularly higher in South Asia due to frequent exposure to risk factors such as betel quid chewing, smoking, and alcohol consumption [21, 22]. Sri Lanka has the eighth highest oral cancer mortality rate in the world. Lip and oral cavity cancer is the number one ranked malignancy among men and $5^{\text {th }}$ in women in Sri Lanka $[1,23]$.

More than 90 out of 100 (90\%) mouth and oropharyngeal cancers are squamous cell carcinomas [24]. Even though the disease burden is as per the situation described earlier, a very minimal number of studies have been carried out in Sri Lanka to identify the connection between histopathological and molecular parameter expressions with prognosis of OSCC. Three main categories such as patient, tumour, and treatment-related factors are important in predicting the prognosis of OSCC [3]. Sex and age, tobacco and alcohol, socioeconomic conditions, and diagnostic delays were identified as patient-related factors while anatomic site, disease staging, cervical node metastases, depth of invasion, extracapsular spread, histological differentiation, and molecular markers were described as tumour-related factors. 
Therefore, we focused on some of the patient- and tumour-related factors, such as demographic data, anatomic site, tumour stage, lymph node metastasis, POI, histological grading, and expression of selected molecular markers. Although many studies have attempted to analyse various combinations of these factors, a model with reliable practical implications for the management of OSCC is yet to be developed [25-27].

\subsection{Distribution of Demographic Data. According to national} cancer incidence data 2014, the highest overall cancer incidence was recorded from the age category 70-74 years with 571.7 per 100,000 in Sri Lanka [1]. In a study on independent prognostic factors of 861 cases of OSCC in Korean adults, a multivariate Cox regression analysis has shown that age, gender, composite stage, and treatment method were significant independent prognostic factors [28]. Oral cancer is usually a disease that occurs in males after the 5th decade of life [29]. Coinciding with this observation, in the current study, out of a sample of 290, the highest number of patients was from the 51-60-year category. But age was not identified as an independent prognostic factor. However, a retrospective study in Karachi, Pakistan, had $77.9 \%$ (out of 80 OSCCs) within the 31-60-year age group [30].

The lip, oral cavity, and pharynx are the leading cancer sites for Sri Lankan males (24\% of all cancer sites). According to national cancer statistics, 1888 cases were reported in males, while 534 cases among the females [1]. Therefore, the average male to female ratio of oral cancer is $3.5: 1$. According to a study on epidemiology and aetiology of OSCC worldwide, females are less commonly affected by oral cancer, largely reflecting greater use of relevant habits by men $[29,31]$. Our results were similar to these findings with $75.2 \%$ cases in males (male : female $=3: 1$ ), confirming the predominance of OSCC in males in Sri Lanka. The prevalence of OSCC due to HPV infection has caused a change in gender distribution [32].

4.2. Clinical and Histopathological Parameters. According to the studies by Mirza D et al. [30] and Mirza S et al. [33], buccal mucosa was encountered with the highest percentage of OSCC (50\% and 46.8\%, respectively). Another study also showed that most tumours were seen in the buccal cavity (54\%) followed by the tongue (24\%) [34]. In contrast, a Brazilian study showed that $37 \%$ of cases out of 346 were from the lateral border of the tongue [35]. In an Indian study with 295 OSCC cases, the most common site was the mandibular alveolus region [36]. In the current study, the buccal mucosa was the most common primary site in both genders. However Sahaf et al. reported that majority of females $(41.7 \%)$ in their study had SCC in the tongue, while majority of males (33.3\%) had SCC in the buccal mucosa [31]. Hence, it is evident that the most common site of OSCC changes from population to population. The main reason for this site predilection in different regions is due to risk habits. In countries in South Asia where betel quid chewing is common, the frequent site of occurrence is the buccal mucosa as they keep the quid in the buccal pouch. In Western countries where alcohol and smoking are common practices, the frequent sites of involvement are the tongue and floor of the mouth. Apart from these main habits, factors such as genetic predisposition, viruses, and radiation can be considered as other reasons for this difference.

Early detection of cancer is very vital in the prognosis of patients identified with OSCC. The survival rates highly rely on the stage of the tumour at diagnosis. $57.7 \%$ of the cases in this study presented to clinicians at stage 4 and only $5.2 \%$ at stage 1 . Mirza et al. also indicated that their stage 4 and stage 1 presentations were $50.7 \%$ and $14.7 \%$, respectively, and they found a significant association with stage and metastasis [33]. In an Indian study, majority of the patients $(243,82.37 \%)$ were presented at stage 3 and none at stage 1 [36]. Another study in Sri Lanka, where 193 previously diagnosed OSCC patients were followed up for 5 years, found that the tumour stage has a significant association with the 5-year survival $(p<0.05)$ [6]. The current study found a statistically significant association between stage and metastasis $(p<0.05)$.

A positive significant correlation between the host response and metastasis of OSCC was found by logistic regression analysis. Our above results are in agreement with the findings by de Matos et al. [37]. In a study by Chatzistamou et al. [38] on oral tongue SCC, high-density inflammatory host response was correlated with a favourable survival. Lundqvist et al. [39] found a correlation between density of host response and favourable response to radiotherapy in tongue SCC. These results were in line with our study, where a statistically significant association between the 3-year survival rate and host response was revealed $(p<0.05)$. Therefore, it can be assumed that the antitumour characteristics of inflammatory cells might be a reason for better prognosis of patients.

In this study, most of the tumours were well differentiated $(50.3 \%)$ similar to the findings by Sahaf et al. [31] where $68.4 \%$ of tumours were well differentiated. However, Lundqvist et al. [39] and Mirza et al. [33] showed in their studies that the majority were moderately differentiated tumours (40\% and $62.1 \%$, respectively). Logistic regression analysis showed a positive relationship between metastasis and tumour differentiation where metastatic rate was increased when tumour differentiation varied from well to poor. In a study done by Dissanayaka et al. [6], degree of differentiation was well correlated with the five-year survival rate. Further, it is advisable to include level of differentiation to multivariable models of survival as a covariate to improve prognostic accuracy [40].

The cervical lymph node status is considered the most important prognostic factor in head and neck cancer. According to a study by Woolgar et al. [41], microscopic extracapsular spread is of critical importance and it should be incorporated into pathological staging systems. In the current study, a statistically significant relationship was found between metastasis and 5-year and 3-year survival rates $(p<0.05)$. Some studies showed a significant association between POI and metastasis [42, 43], and this study also revealed the same.

POI has been identified as one of the most reliable predictive factors of OSCC. POI type IV where there is an individual tumour cell invading the host tissues at the tumour front 
suggests poor prognosis [6]. Majority of the patients who did not survive for 3 years and 5 years had POI type IV $(p<0.05)$, and many other studies supported this finding [44-46]. Furthermore, our study showed that most $(70.5 \%)$ of the POI type IV was from stage 4 cases and may be served as a contributory factor for poor prognosis. However, the available literature has not analysed the above relationships. Extracapsular spread and POI showed a positive correlation. Majority of the younger patients in the present study also had POI type IV thus leading to poor prognosis.

The time span to distinguish the lesion as a recurrence (local recurrence/nodal recurrence) has not been welldescribed. Camisasca et al. [47] have demonstrated that poorly differentiated tumours located in the tongue and showing POI type III or IV are associated with higher recurrence. Majority of the cases that presented with recurrence in our study were from POI category IV $(p<0.05)$. According to the study carried out by Brandwein-Gensler et al. [48], the traditional POI in the Bryne et al. [49] multifactorial grading system at the tumour front has been modified by adding a pattern $\mathrm{V}$ which is defined as tumour satellites, and this parameter is identified as worst POI (WPOI) at the tumour/host interface. This WPOI has been identified as an independent predictor of local recurrence and overall survival. Recurrence is considered the most common cause of treatment failure and is usually attributed to incomplete surgical resection of the tumour [50]. The highest recurrence percentage of $75.0 \%$ was reported from the age $31-40$-year category in the current study. This confirms that the presence of type IV POI in the younger group leads to high recurrence, hence poor prognosis with reduced 5-year survival rate.

Survival of the cancer patient is the main prognostic indicator of OSCC. Despite recent advances in treatment, the 5year survival rate of OSCC still remains at 50\% [51]. Locoregional recurrence has been identified as an important factor that affects survival [48]. Based on the Kaplan-Meier survival analysis, Yanamoto et al. [46] revealed similar results to current findings. In their study, 5-year cancer-specific survival rate was $92 \%$ in the nonrecurrence group and $30 \%$ in the recurrence group ( $p<0.0001, \log$-rank test). Comparatively, Wang et al. [52] using the Kaplan-Meier method and logrank test showed that 2-year and 5-year survival rates were lower in patients with recurrence than in those without recurrence $(67.6 \%$ vs. $88.0 \%, 31.8 \%$ vs. $79.9 \%, p<0.001)$. Camisasca et al. [47] also has shown that local recurrence is a significant prognostic factor for 5-year disease-specific survival similar to our study.

4.3. Expression of Molecular Markers. The effects of activation of EGFR in keratinocytes include induction of cellular proliferation, reduction in differentiation, induction of migration of normal keratinocytes, and inhibition of apoptosis while increasing survival of keratinocytes. Hiraishi et al. [53] have shown that out of 52 patients with OSCC, 33 had high EGFR expression immunohistochemically (63.4\%), and a study on tissue microarray-based IHC by Laimer et al. [54] showed similar results with high EGFR expression of $73.42 \%$ of the tumour sample. Statistically significant relationship between lymph node metastasis and survival rates with higher expression of EGFR was identified in the present study $(p<0.05)$. A multivariate analysis by Laimer et al. identified EGFR overexpression as an independent prognostic marker $(p=0.02)$ [54] similar to our study. However, the study by Kudo et al. [55] did not find a positive correlation by Fisher's exact test. This is the first study comparing POI and EGFR expression, and we found a significant association $(p<0.05)$. However, Ang et al. [56] has revealed that immunohistochemical EGFR expression was a strong independent prognostic indicator for overall survival and disease-free survival through a multivariate analysis performed using Cox proportional hazard model. Based on these results, they have suggested that the patients who have EGFR-positive immunoexpression in their OSCCs should be considered for more aggressive combined therapies or enrolment into trials targeting EGFR signalling pathways.

In the present study, EGFR and MMP-9 were found as independent predictive variables in the logistic regression analysis.

Many cancers have been related with the overexpression and mutations of $\beta$-catenin. Abnormalities of cell adhesion molecules are known to play an important role in invasion and metastasis of cancer cells through loss of cell-to-cell adhesion [55]. A study done by Kudo et al. [55] suggests that the invasion and metastasis of OSCC cells require methylation of E-cadherin and/or degradation of membranous $\beta$ catenin. In a study with 30 OSCC samples, [57] suggested that the use of loss of expression of $\beta$-catenin as a marker (by IHC) of nodal metastasis in OSCC is unreliable (Fisher's exact test). However, they have found that loss of expression of $\beta$-catenin has an association with lower degree of differentiation. Another study using 24 OSCCs showed that there is no difference between the expression of $\beta$-catenin in metastatic and nonmetastatic groups through nonparametric MannWhitney $U$ test for exploratory descriptive analysis [58]. Contrasting to these outcomes, Tanaka et al. [16] have found significantly greater reduction in expression level of $\beta$ catenin in the lymph node metastatic group $(n=64)$ compared to the nonmetastatic group $(n=95)(p=0.001)$ in a study on 159 OSCC samples, using Kaplan-Meier method log-rank test for statistical analysis. Although the results are not significant, more metastasis was seen in the negative $\beta$ catenin expression group.

HIF- $1 \alpha$ is a molecule that is mainly activated under hypoxic conditions and has been proposed to play a role in malignant transformation of OSF and may be possible to use as a marker $[10,11]$. Santos et al. [59] have demonstrated by IHC on 66 tissue microarrays that high HIF- $1 \alpha$ expression was associated with local disease-free survival $(p=0.013)$ through chi-square and Fisher exact test and multivariate logistic regression. And also, a multivariate Cox regression analysis suggested that HIF- $1 \alpha$ is an independent prognostic marker correlating with a disease-specific survival $(p=0.001)$ [60] by IHC on 82 OSCC cases. In the current study, no statistically significant relationship was found between HIF- $1 \alpha$ and metastasis. However, highly expressed HIF-1 $\alpha$ cases were from the POI type IV revealing a statistically significant relationship $(p<0.05)$. Although we were unable to find studies connecting HIF-1 $\alpha$ expression with POI, the 
relationship we revealed may show an indirect association between HIF- $1 \alpha$ expression and lymph node metastasis as POI type IV is significantly associated with lymph node metastasis. Therefore, this molecule may have a role as a prognostic marker in OSCC.

Periostin is a secreted protein that stimulates metastatic growth by promoting cancer cell survival, invasion, and angiogenesis; thus, it can be a useful marker to predict the behaviour of cancer (Kudo et al., 2007). In the current study, periostin was strongly expressed in $64.5 \%$ of the cases, and yet, we could not demonstrate a statistically significant relationship between periostin expression and metastasis.

Lymphangiogenesis is reported to be induced by vascular endothelial growth factor-C (VEGF-C). This molecule promotes tumour invasion and metastasis. The density of microvessels is increased by VEGF-C influencing the metastatic behaviour of the tumour. In an IHC study, Sedivy et al. [15] concluded that VEGF-C expression in OSCC triggers lymphatic angiogenesis, which may result in a higher risk for cervical lymph node metastasis. Kudo et al. [61] also have identified that periostin expression would induce upregulation of VEGF-C, which may in turn promote lymphangiogenesis. Although majority of high VEGF-C expression cases were from the POI type IV, a statistically significant association was not identified between these two parameters as in the study done by Siriwardena et al. [14]. The current study also did not identify a statistically significant relationship. In contrast to our study, Kishimoto et al. revealed a significant relationship between VEGF-C expression and lymph node metastasis $(p<0.001)$ with Fisher's exact test [62].

Matrix metalloproteinase-9 (MMP-9) is a protein from the matrix metalloproteinase family which plays many roles in the normal physiological processes. Although its role is not clear in cancer, many studies have reported the importance of MMP-9 expression in OSCC [20, 63, 64]. Zhou et al. [64] found a significant difference between MMP-9 overexpression and nodal metastasis $(p<0.01)$. A study done by Ruokolainen et al. [20] showed that MMP-9 expression (IHC) was statistically correlated with cause-specific survival by Kaplan-Meier analysis $(p=0.013)$. Hong et al. also supported the same [63]. However, a statistically significant association was shown between MMP-9 expression and POI in the current study, hence metastasis.

4.4. Logistic Regression Model in Multivariate Analysis. When considering the factors that influence metastasis in the current study, logistic regression analysis has been used to specify positive and negative parameters. According to the results, POI and differentiation of the tumour are independent prognostic markers for lymph node metastasis. Although some of the previous studies have discovered a correlation between metastasis of OSCC and some molecular markers $[5,12,16,53,63,65]$, we found that EGFR shows a positive correlation. Moreover, none of these studies include a sample size as large as the current study.

High expression of EGFR, HIF- $1 \alpha$, periostin, and MMP9 and loss of expression in $\beta$-catenin showed a direct relationship with pattern of invasion. As there is a significant relationship between POI with lymph node metastasis and survival, it may indirectly relate the above molecules with lymph node metastasis and survival.

A study with a cohort of 193 patients has revealed tumour stage, POI, and excision margins to be the most predictive factors of survival in OSCC [6]. Pattern of invasion was identified as the best prognosticator in the stepwise Cox regression model [65]. Yuen et al. indicated that tumour thickness was the only significant prognostic factor for subclinical nodal metastasis in a multivariate logistic regression analysis on stage I and II oral tongue carcinomas [66]. They also have shown that both tumour thickness and perineural infiltration were significant independent prognostic factors of local recurrence.

\section{Conclusions}

Three-year and 5-year survival rates were significantly associated with lymph node metastasis and recurrence. POI was significantly correlated with tumour size, stage, 3-year survival, and all the tested molecules. POI, level of differentiation, and expression of EGFR are independent prognostic markers for lymph node metastasis. Therefore, these parameters may help in treatment planning of the clinically negative neck.

\section{Data Availability}

All data included here. Raw data can be provided upon request.

\section{Conflicts of Interest}

The authors declare that they have no conflicts of interest.

\section{Acknowledgments}

The authors received funding from the National Research Council Sri Lanka.

\section{References}

[1] Sri Lanka National Cancer Control Program, Cancer incidence data Sri Lanka 2014, Government Cancer Institute, Ministry of Health, Sri Lanka, 2014.

[2] A. Capote, V. Escorial, M. F. Muñoz-Guerra, F. J. RodríguezCampo, C. Gamallo, and L. Naval, "Elective neck dissection in early-stage oral squamous cell carcinoma-does it influence recurrence and survival?," Head \& Neck, vol. 29, no. 1, pp. 311, 2007.

[3] J. Massano, F. S. Regateiro, G. Januário, and A. Ferreira, "Oral squamous cell carcinoma: review of prognostic and predictive factors," Oral Surgery, Oral Medicine, Oral Pathology, Oral Radiology, and Endodontology, vol. 102, no. 1, pp. 67-76, 2006.

[4] M. Pentenero, S. Gandolfo, and M. Carrozzo, "Importance of tumor thickness and depth of invasion in nodal involvement and prognosis of oral squamous cell carcinoma: a review of the literature," Head \& Neck, vol. 27, no. 12, pp. 1080-1091, 2005.

[5] B. S. M. S. Siriwardena, A. Tilakaratne, E. A. P. D. Amaratunga et al., "Analysis of histopathological and immunohistochemical differences of oral squamous cell carcinoma in young and old 
patients in Sri Lanka," Journal of Oral Pathology \& Medicine, vol. 36, no. 6, pp. 357-362, 2007.

[6] W. L. Dissanayaka, G. Pitiyage, P. V. Kumarasiri, R. L. Liyanage, K. D. Dias, and W. M. Tilakaratne, "Clinical and histopathologic parameters in survival of oral squamous cell carcinoma," Oral surgery, oral medicine, oral pathology and oral radiology, vol. 113, no. 4, pp. 518-525, 2012.

[7] H. Schliephake, "Prognostic relevance of molecular markers of oral cancer-a review," International Journal of Oral and Maxillofacial Surgery, vol. 32, no. 3, pp. 233-245, 2003.

[8] M. Bryne, "Is the invasive front of an oral carcinoma the most important area for prognostication?," Oral Diseases, vol. 4, no. 2, pp. 70-77, 1998.

[9] G. Cortesina and T. Martone, "Molecular metastases markers in head and neck squamous cell carcinoma: review of the literature," Actaotorhinolaryngologicaitalica, vol. 26, no. 6, p. 317, 2006.

[10] W. M. Tilakaratne and E. H. Nissanka-Jayasuriya, "Value of HIF- $1 \alpha$ as an independent prognostic indicator in oral squamous cell carcinoma," Expert Review of Molecular Diagnostics, vol. 11, no. 2, pp. 145-147, 2014.

[11] W. M. Tilakaratne, Z. Iqbal, M. T. Teh et al., "Upregulation of HIF- $1 \alpha$ in malignant transformation of oral submucous fibrosis," Journal of Oral Pathology \& Medicine, vol. 37, no. 6, pp. 372-377, 2008.

[12] B. S. M. S. Siriwardena, Y. Kudo, I. Ogawa et al., "Periostin is frequently overexpressed and enhances invasion and angiogenesis in oral cancer," British Journal of Cancer, vol. 95, no. 10, pp. 1396-1403, 2006.

[13] E. M. Deraz, Y. Kudo, M. Yoshida et al., "MMP-10/stromelysin-2 promotes invasion of head and neck cancer," PLoS One, vol. 6, no. 10, article e25438, 2011.

[14] B. S. M. S. Siriwardena, Y. Kudo, I. Ogawa, M. N. G. P. K. Udagama, W. M. Tilakaratne, and T. Takata, "VEGF-C is associated with lymphatic status and invasion in oral cancer," Journal of Clinical Pathology, vol. 61, no. 1, pp. 103-108, 2008.

[15] R. Sedivy, J. Beck-Mannagetta, C. Haverkampf, W. Battistutti, and S. Hönigschnabl, "Expression of vascular endothelial growth factor-C correlates with the lymphatic microvessel density and the nodal status in oral squamous cell cancer," Journal of Oral Pathology \& Medicine, vol. 32, no. 8, pp. 455-460, 2003.

[16] N. Tanaka, T. Odajima, K. Ogi, T. Ikeda, and M. Satoh, "Expression of E-cadherin, $\alpha$-catenin, and $\beta$-catenin in the process of lymph node metastasis in oral squamous cell carcinoma," British Journal of Cancer, vol. 89, no. 3, pp. 557$563,2003$.

[17] G. R. Thomas, H. Nadiminti, and J. Regalado, "Molecular predictors of clinical outcome in patients with head and neck squamous cell carcinoma," International Journal of Experimental Pathology, vol. 86, no. 6, pp. 347-363, 2005.

[18] L. R. Oliveira and A. Ribeiro-Silva, "Prognostic significance of immunohistochemical biomarkers in oral squamous cell carcinoma," International Journal of Oral and Maxillofacial Surgery, vol. 40, no. 3, pp. 298-307, 2011.

[19] J. A. C. Hanemann, D. T. Oliveira, S. Nonogaki et al., "Expression of E-cadherin and $\beta$-catenin in basaloid and conventional squamous cell carcinoma of the oral cavity: are potential prognostic markers?," BMC cancer, vol. 14, no. 1, p. 1, 2014.

[20] H. Ruokolainen, P. Pääkkö, and T. Turpeenniemi-Hujanen, "Expression of matrix metalloproteinase-9 in head and neck squamous cell carcinoma a potential marker for prognosis," Clinical Cancer Research, vol. 10, no. 9, pp. 3110-3116, 2004.

[21] WHO programmes, Cancer, oral cancer, early diagnosis and screening, 2020, http://www.who.int/cancer/prevention/ diagnosis-screening/oral-cancer/en/.

[22] WHO, The world oral health report, World Health Organization, Geneva, 2003.

[23] Globocan, Cancer data of Sri Lanka, IADR, 2018.

[24] National Cancer Registration and Analysis Service (NCRAS), Cancer statistics: availability and location, PHE publications, 2017.

[25] R. K. D. Silva, B. S. M. S. Siriwardena, A. Samaranayaka, W. A. M. U. L. Abeyasinghe, and W. M. Tilakaratne, "A model to predict nodal metastasis in patients with oral squamous cell carcinoma," PLoS One, vol. 13, no. 8, article e0201755, 2018.

[26] B. S. M. S. Siriwardena, R. M. S. G. K. Rasnayaka, Y. Masood, M. Masood, P. V. R. Kumarasiri, and W. M. Tilakaratne, "A predictive model of oral cancer metastasis for different cancer sites and age groups," Journal of Investigative and Clinical Dentistry, vol. 6, pp. 1-5, 2015.

[27] B. S. M. S. Siriwardena, I. K. Rambukewela, T. N. Pitakotuwage, M. N. G. P. K. Udagama, P. V. R. Kumarasiri, and W. M. Tilakaratne, "A predictive model to determine the pattern of nodal metastasis in oral squamous cell carcinoma," BioMed Research International, vol. 2018, Article ID 8925818, 7 pages, 2018.

[28] K. K. Choi, M. J. Kim, P. Y. Yun et al., "Independent prognostic factors of 861 cases of oral squamous cell carcinoma in Korean adults," Oral Oncology, vol. 42, no. 2, pp. 208-217, 2006.

[29] N. W. Johnson, P. Jayasekara, and H. K. Amarasinghe, "Squamous cell carcinoma and precursor lesions of the oral cavity: epidemiology and aetiology," Periodontology, vol. 57, no. 1, pp. 19-37, 2000.

[30] D. Mirza, G. Raza, A. Basit, K. Naqvi, S. Ahmed, and Z. A. Abassi, "Oral squamous cell carcinoma (OSCC) in Karachi city," Pakistan Oral \& Dental Journal, vol. 36, no. 3, pp. 391394, 2016.

[31] R. Sahaf, N. Naseem, R. Anjum, and A. Nagi, "Oral squamous cell carcinoma: a clinicopathologic study," Pakistan Oral \& Dental Journal, vol. 37, no. 1, pp. 49-54, 2017.

[32] L. M. Brown, D. P. Check, and S. S. Devesa, "Oral cavity and pharynx cancer incidence trends by subsite in the United States: changing gender patterns," Journal of Oncology, vol. 2012, Article ID 649498, 10 pages, 2012.

[33] S. Mirza, N. I. Hadi, S. Akram, N. Wahab, and Z. Akram, "Histopathological predictors of nodal metastases in oral squamous cell carcinoma," Pakistan journal of Medicine and Dentistry, vol. 5, no. 3, pp. 12-16, 2016.

[34] S. Akram, T. Mirza, M. A. Mirza, and M. Qureshi, "Emerging patterns in clinico-pathological spectrum of oral cancers," Pakistan journal of medical sciences, vol. 29, no. 3, p. 783, 2013.

[35] F. R. Pires, A. B. Ramos, J. B. Oliveira, A. S. Tavares, P. S. Luz, and T. C. Santos, "Oral squamous cell carcinoma: clinicopathological features from 346 cases from a single oral pathology service during an 8-year period," Journal of Applied Oral Science, vol. 21, no. 5, pp. 460-467, 2013.

[36] R. Shenoi, V. Devrukhkar, B. K. Sharma, S. B. Sapre, and A. Chikhale, "Demographic and clinical profile of oral squamous cell carcinoma patients: a retrospective study," Indian journal of cancer, vol. 49, no. 1, p. 21, 2012. 
[37] F. R. de Matos, L. E. D. de Araújo, L. M. Queiroz, and É. J. da Silveira, "Analysis of inflammatory infiltrate, perineural invasion, and risk score can indicate concurrent metastasis in squamous cell carcinoma of the tongue," Journal of Oral and Maxillofacial Surgery, vol. 70, no. 7, pp. 1703-1710, 2012.

[38] I. Chatzistamou, J. Rodriguez, T. Jouffroy et al., "Prognostic significance of tumor shape and stromal chronic inflammatory infiltration in squamous cell carcinomas of the oral tongue," Journal of Oral Pathology \& Medicine, vol. 39, no. 9, pp. 667-671, 2010.

[39] L. Lundqvist, H. Stenlund, G. Laurell, and K. Nylander, "The importance of stromal inflammation in squamous cell carcinoma of the tongue," Journal of Oral Pathology \& Medicine, 1, vol. 41, no. 5, pp. 379-383, 2012.

[40] B. Thomas, M. Stedman, and L. Davies, "Grade as a prognostic factor in oral squamous cell carcinoma: a population-based analysis of the data," The Laryngoscope, vol. 124, no. 3, pp. 688-694, 2014.

[41] J. A. Woolgar, S. N. Rogers, D. Lowe, J. S. Brown, and E. D. Vaughan, "Cervical lymph node metastasis in oral cancer: the importance of even microscopic extracapsular spread," Oral Oncology, vol. 39, no. 2, pp. 130-137, 2003.

[42] T. Khwaja, A. S. Tayaar, S. Acharya, J. Bhushan, and M. V. Muddapur, "Pattern of invasion as a factor in determining lymph node metastasis in oral squamous cell carcinoma," The Journal of Cancer Research and Therapeutics, vol. 14, no. 2, p. 382, 2017.

[43] A. Alkaisi, H. Zaidan, and I. A. A. Kabtan, "The predictive value of tumor depth for cervical lymph node metastasis in oral squamous cell carcinoma; prospective and retrospective study in Iraq," Journal of Cancer Science and Therapy, vol. 6, pp. 253-257, 2014.

[44] M. Julaha, B. Hazarika, U. Mukherjee, and H. Chaturvedi, "O28. Tumour depth and pattern of invasion: there relationship to neck nodal metastasis in oral cavity squamous cell carcinomas," Oral Oncology, vol. 47, p. S37, 2011.

[45] T. M. Søland, I. J. Brusevold, H. S. Koppang, K. Schenck, and M. Bryne, "Nerve growth factor receptor (p75NTR) and pattern of invasion predict poor prognosis in oral squamous cell carcinoma," Histopathology, vol. 53, no. 1, pp. 62-72, 2008.

[46] S. Yanamoto, S. I. Yamada, H. Takahashi et al., "Predictors of locoregional recurrence in T1-2N0 tongue cancer patients," Pathology \& Oncology Research, vol. 19, no. 4, pp. 795-803, 2013.

[47] D. R. Camisasca, M. A. Silami, J. Honorato, F. L. Dias, P. Faria, and S. Lourenço, "Oral squamous cell carcinoma: clinicopathological features in patients with and without recurrence," Oto-Rhino-Laryngology, Head and Neck Surgery, vol. 73, no. 3, pp. 170-176, 2011.

[48] M. Brandwein-Gensler, M. S. Teixeira, C. M. Lewis et al., "Oral squamous cell carcinoma," American Journal of Surgical Pathology, vol. 29, no. 2, pp. 167-178, 2005.

[49] M. Bryne, H. S. Koppang, R. Lilleng, and A. Kjærheim, "Malignancy grading of the deep invasive margins of oral squamous cell carcinomas has high prognostic value," The Journal of Pathology, vol. 166, no. 4, pp. 375-381, 1992.

[50] A. Almangush, Histopathological predictors of early stage oral tongue cancer, Finnish Doctoral Program in Oral Sciences, Faculty of Medicine, University of Helsinki, Finland, 2015.

[51] Oral cancer facts, Understanding, The oral cancer foundation, HomeMay 2014, http://www.oralcancerfoundation.org/ erstanding/.
[52] B. Wang, S. Zhang, K. Yue, and X. D. Wang, "The recurrence and survival of oral squamous cell carcinoma: a report of 275 cases," Chinese journal of cancer, vol. 32, no. 11, p. 614, 2013.

[53] Y. Hiraishi, T. Wada, K. Nakatani, K. Negoro, and S. Fujita, "Immunohistochemical expression of EGFR and p-EGFR in oral squamous cell carcinomas," Pathology \& Oncology Research, vol. 12, no. 2, pp. 87-91, 2006.

[54] K. Laimer, G. Spizzo, G. Gastl et al., "High EGFR expression predicts poor prognosis in patients with squamous cell carcinoma of the oral cavity and oropharynx: a TMA-based immunohistochemical analysis," Oral Oncology, vol. 43, no. 2, pp. 193-198, 2007.

[55] Y. Kudo, B. S. M. S. Siriwardena, ,H. Hatano, I. Ogawa, and T. Takata, "Periostin: novel diagnostic and therapeutic target for cancer," Histology and histopathology, vol. 22, no. 10, pp. 1167-1174, 2007.

[56] K. K. Ang, B. A. Berkey, X. Tu et al., "Impact of epidermal growth factor receptor expression on survival and pattern of relapse in patients with advanced head and neck carcinoma," Cancer Research, vol. 62, no. 24, pp. 7350-7356, 2002.

[57] F. Mahomed, M. Altini, and S. Meer, "Altered E-cadherin/ $\beta$ catenin expression in oral squamous carcinoma with and without nodal metastasis," Oral Diseases, vol. 13, no. 4, pp. 386392, 2007.

[58] R. D. A. Freitas, E. J. D. Silveira, J. P. B. D. Silveira, F. M. D. Silva, and R. F. B. D. Amorim, "Correlation of $\beta$-catenin expresssion and metastasis in tongue squamous cell carcinoma," Acta Cirurgica Brasileira, vol. 25, no. 6, pp. 513-517, 2010.

[59] M. dos Santos, A. M. da Cunha Mercante, I. D. Louro et al., "Hif 1 alpha expression predicts survival of patients with squamous cell carcinoma of the oral cavity," PLOS ONE, vol. 7, no. 9, article E45228, 2012.

[60] A. W. Eckert, A. Schutze, M. H. W. Lautner, H. Taubert, J. Schubert, and U. Bilkenroth, "HIF-1a is a prognostic marker in OSCC," International Journal of Biological Markers, vol. 25, no. 2, pp. 87-92, 2018.

[61] Y. Kudo, S. Iizuka, M. Yoshida et al., "Periostin directly and indirectly promotes tumor lymphangiogenesis of head and neck cancer," PLoS One, vol. 7, no. 8, article e44488, 2012.

[62] K. Kishimoto, A. Sasaki, Y. Yoshihama, H. Mese, G. Tsukamoto, and T. Matsumura, "Expression of vascular endothelial growth factor- $\mathrm{C}$ predicts regional lymph node metastasis in early oral squamous cell carcinoma," Oral Oncology, vol. 39, no. 4, pp. 391-396, 2003.

[63] S. D. Hong, S. P. Hong, J. I. Lee, and C. Y. Lim, "Expression of matrix metalloproteinase- 2 and -9 in oral squamous cell carcinomas with regard to the metastatic potential," Oral Oncology, vol. 36, no. 2, pp. 207-213, 2000.

[64] C. X. Zhou, Y. Gao, N. W. Johnson, and J. Gao, "Immunoexpression of matrix metalloproteinase-2 and matrix metalloproteinase- 9 in the metastasis of squamous cell carcinoma of the human tongue," Australian dental journal, vol. 55, no. 4, pp. 385-389, 2010.

[65] T. Kurokawa, M. Miyamoto, K. Kato et al., "Overexpression of hypoxia-inducible-factor $1 \alpha \quad(\mathrm{HIF}-1 \alpha)$ in oesophageal squamous cell carcinoma correlates with lymph node metastasis and pathologic stage," British Journal of Cancer, vol. 89, no. 6, pp. 1042-1047, 2003.

[66] A. P. W. Yuen, K. Y. Lam, L. K. Lam et al., "Prognostic factors of clinically stage I and II oral tongue carcinoma - a comparative 
study of stage, thickness, shape, growth pattern, invasive front malignancy grading, Martinez-Gimeno score, and pathologic features," Head \& neck, vol. 24, no. 6, pp. 513-520, 2002.

[67] P. Balasundaram, M. K. Singh, A. K. Dinda, A. Thakar, and R. Yadav, "Study of $\beta$-catenin, E-cadherin and vimentin in oral squamous cell carcinoma with and without lymph node metastases," Diagnostic pathology, vol. 9, no. 1, p. 1, 2014.

[68] T. Naruse, G. Kawasaki, S. Yanamoto, A. Mizuno, and M. Umeda, "Immunohistochemical study of VEGF expression in oral squamous cell carcinomas: correlation with the mTOR-HIF-1 $\alpha$ pathway," Anticancer Research, vol. 31, no. 12, pp. 4429-4437, 2011.

[69] E. R. Sauter, M. Nesbit, J. C. Watson, A. Klein-Szanto, S. Litwin, and M. Herlyn, "Vascular endothelial growth factor is a marker of tumor invasion and metastasis in squamous cell carcinomas of the head and neck," Clinical Cancer Research, vol. 5, no. 4, pp. 775-782, 1999. 